\title{
Bronchial epithelial cell B7-1 and B7-2 mRNA expression after lung transplantation: a role in allograft rejection?
}

\author{
A. Elssner*, F. Jaumann*, W-P. Wolf*, M. Schwaiblmair*, J. Behr*, H. Fürst", \\ H. Reichenspurner", J. Briegel ${ }^{+}$, J. Niedermeyer ${ }^{\S}$, C. Vogelmeier*
}

Bronchial epithelial cell B7-1 and B7-2 mRNA expression after lung transplantation: a role in allograft rejection? A. Elssner, F. Jaumann, W-P. Wolf, M. Schwaiblmair, J. Behr, H.Fürst, H. Reichenspurner, J. Briegel, J. Niedermeyer, C. Vogelmeier. (C) ERS Journals Ltd 2002.

ABSTRACT: Obliterative bronchiolitis is commonly interpreted as chronic rejection and involves the bronchial and bronchiolar epithelium. Upregulation of major histocompatibility complex (MHC) II on bronchial epithelial cells (BEC) had been hypothesised to be an important trigger of a bronchus directed rejection response. More recently, the additional expression of the costimulatory molecules B7-1 (CD80) and B7-2 (CD86) on antigen presenting cells were found to play an important role in the activation of T-lymphocytes in transplant rejection. The role of the expression of these molecules by $\mathrm{BEC}$ is unclear.

BEC obtained by bronchial brushing and bronchoalveolar lavage fluid (BALF) cells from lung transplant recipients were studied and evaluated for messenger ribonucleic acid (mRNA) expression of B7-1 and B7-2 by semi-quantitative reverse transcriptasepolymerase chain reaction. Significantly elevated B7-1/glyceraldehyde-3-phosphate dehydrogenase (GAPDH) mRNA ratios were found in BEC from patients examined during the first 3 months after lung transplantation. Interestingly, in a small group of patients with bronchiolitis obliterans syndrome the B7-1/GAPDH and B7-2/GAPDH ratios were significantly elevated for $B E C$, whereas no differences were found for the BALF cells.

In summary, B7 messenger ribonucleic acid expression by bronchial epithelial cells may play a role in (chronic) lung allograft rejection.

Eur Respir J 2002; 20: 165-169.
*Section for Pulmonary Diseases, ${ }^{\#}$ Dep

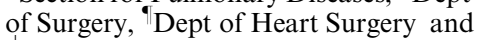
${ }^{+}$Dept of Anaesthesiology, Klinikum Grosshadern, Ludwig-MaximiliansUniversity of Munich, Munich and ${ }^{\S}$ Dept of Internal Medicine, Hannover Medical School, Hannover, Germany.

Correspondence: A. Elssner, Heart and Lung Research Institute, Ohio State University, 473 West 12th Avenue, Columbus, Ohio 43210, USA. Fax: 16142927778

E-mail: elssner-1@medctr.osu.edu

Keywords: B7-1, B7-2, bronchial epithelial cells, bronchiolitis obliterans syndrome, lung transplantation rejection

Received: August 12001

Accepted after revision: February 8 2002

This study was supported by a grant from the Deutsche Forschungsgemeinschaft no. 406/2-1.
In addition to the stimulation induced by the T-cell receptor/major histocompatibility complex (MHC) interaction, costimulatory signals are required for full activation of T-lymphocytes. B7-1 (CD80) and B7-2 (CD86) both serve as costimulatory molecules on the surface of antigen presenting cells (APCs). The B7 signal is transmitted by the counter-receptor CD28 on T-cells [1]. Although B7-1 and B7-2 are typically expressed by "professional" APCs, like macrophages and monocytes, B-lymphocytes, Langerhans' cells in the skin and dendritic cells, expression was also detected on epithelial cells. YE et al. [2] reported B7-1 and B7-2 expression in a human gastric epithelial cell line as well as in isolated epithelial cells from gastric biopsies. Other types of epithelial cells with evidence of B7 expression include ductal and acinar salivary gland epithelial cells from patients with Sjoegren's syndrome [3], biliary epithelial cells from patients suffering from primary biliary cirrhosis or primary sclerosing cholangitis [4], and thyroid follicular cells of surgically-removed thyroid tissue from patients with Hashimoto's thyroiditis [5]. Recently, KANEKO et al. [6] reported B7 expression on bronchiolar and alveolar epithelial cells. The B7 costimulatory pathway is strongly suggested to play an important role in transplant rejection [1]. However, the relevance of B7 molecules in lung transplant (LT) rejection is still unproven. The relevance of the B7 pathway in the pathogenesis of obliterative bronchiolitis $(\mathrm{OB})$ is of particular interest since $\mathrm{OB}$ represents the most important complication in the long-term follow-up after LT regarding prevalence, morbidity, and mortality [7]. The aim of this study was to examine LT recipients for the presence of B7-1 and B7-2 messenger ribonucleic acid (mRNA) in cells from bronchial brush biopsies and from bronchoalveolar lavage fluid (BALF) cells.

\section{Materials and methods}

\section{Patients}

Patients who underwent lung transplantation were studied in intervals of 3-6 months by lung function testing, bronchoalveolar lavage and bronchial brush biopsies. The patients were stratified into three groups: early after LT ( $<3$ months) $(n=7)$; patients with bronchiolitis obliterans syndrome (BOS) $(n=4)$; and patients without obvious allograft dysfunction 
after LT (n=8) (table 1$)$. Only clinically stable patients without evidence of acute graft rejection and signs of infection (including microbiological analysis of BALF) were eligible. Routine lung biopsies were not performed at the time of bronchoalveolar lavage. The diagnosis BOS was established by evaluating the results from lung function testing according to the criteria of the Registry of the International Society for Heart and Lung Transplantation [8], with the following exception. In the BOS group, three of the four patients had a forced expiratory volume in one second (FEV1) $>80 \%$ and $\leqslant 90 \%$ of baseline but a forced midexpiratory flow (FEF25\%-75\%) $<80 \%$ of baseline following reports that the $\mathrm{FEF} 25 \%-75 \%$ may be a more sensitive marker for the detection of developing BOS [9].

\section{Bronchoalveolar lavage}

Bronchoalveolar lavage was performed using a flexible bronchoscope passed to a wedged position into three different lobes, followed by instillation of five aliquots of $20 \mathrm{~mL}$ of $0.9 \% \mathrm{NaCl}$ into each lobe (300 mL total). In several patients, smaller volumes (100-200 mL total) had to be used because of reduced respiratory function. The cells were stored at $-70^{\circ} \mathrm{C}$ until ribonucleic acid (RNA) extraction was performed as described below.

\section{Bronchial brush specimens}

To obtain cells from the airway mucosa, a sheathed bronchial specimen brush (Product \# 149R; Mill Rose Laboratories, Mentor, OH, USA) was advanced through the operating channel of the bronchoscope, positioned in a segment bronchus, and pushed gently forward and back. After retracting the tip into the protective sheath the brush was removed. In order to harvest a sufficient number of cells, this procedure was repeated up to five times. The cells were gently removed from the brush by light shaking in saline solution and subsequently stored at $-70^{\circ} \mathrm{C}$. Total cell counts were measured by a Coulter counter (Coulter Electronics, Hialeah, FL, USA). A cell differential of each sample was obtained from slide preparations, which were stained with May-Grünwald-Giemsa.

\section{Semiquantitative polymerase chain reaction}

Frozen epithelial cells were lysed, the RNA was extracted and they were reverse transcribed into complementary deoxyribonucleic acid. The following specific primer sets were used for the amplification of glyceraldehyde-3-phosphate dehydrogenase (GAPDH; housekeeping gene used as a reference), B7-1 and B7-2. GAPDH: Forward: 5'-TGAAGGTCGGAGTCAACGGATTTGGT-3'; Reverse: 5'-CATGTGGGCCATGAGGTCCACCAC-3' (size of polymerase chain reaction (PCR) product: 900 base pairs (bp)). B7-1: Forward: 5'-AGTACAAGAACCGGACCATC3'; Reverse: 5'-GGCGTACACTTTCCCTTCTC-3' (size of PCR product: $605 \mathrm{bp}$ ). B7-2: Forward: 5' AGGACAAGGGCTTGTATCAA-3'; Reverse: 5' ATTGCTCGTAACATCAGGGA-3' (size of PCR product: $332 \mathrm{bp}$ ). The cycle conditions for GAPDH were: $94^{\circ} \mathrm{C}$ for $3 \mathrm{~min} / 94^{\circ} \mathrm{C}$ for $45 \mathrm{~s} / 60^{\circ} \mathrm{C}$ for $45 \mathrm{~s} / 72^{\circ} \mathrm{C}$ for $1 \mathrm{~min}$ for 24 cycles, followed by an extension step of $10 \mathrm{~min}$ at $72^{\circ} \mathrm{C}$. The same cycle conditions were used for B7-1 and B7-2. The annealing temperature and PCR cycles for $\mathrm{B} 7-1$ and $\mathrm{B} 7-2$ were $60^{\circ} \mathrm{C}$ and 40 cycles, respectively. In preceding experiments

Table 1. - Clinical status of the patients

\begin{tabular}{|c|c|c|c|}
\hline & $<3$ months post $\mathrm{LT}$ & BOS & non-BOS \\
\hline Patients $n$ & 7 & 4 & 8 \\
\hline Age on the day of bronchoscopy & $41 \pm 3$ & $43 \pm 8$ & $42 \pm 5$ \\
\hline Time since transplant months & $1.4 \pm 0.3$ & $23.3 \pm 8.6$ & $15.9 \pm 2.3$ \\
\hline Sex F:M & $6: 1$ & $1: 3$ & $3: 5$ \\
\hline \multicolumn{4}{|l|}{ Underlying disease $n$} \\
\hline Emphysema & 2 & 2 & 3 \\
\hline Pulmonary fibrosis & 5 & 1 & 2 \\
\hline Cystic fibrosis & & 1 & 1 \\
\hline Primary pulmonary hypertension & & & 1 \\
\hline Lymphangioleiomyomatosis & & & 1 \\
\hline \multicolumn{4}{|l|}{ Type of transplant $n$} \\
\hline Single lung & 6 & 3 & 4 \\
\hline Double lung & 1 & 1 & 3 \\
\hline Heart and lung & & & 1 \\
\hline FEV1 before bronchoscopy $\%$ of baseline & $99.3 \pm 0.6$ & $76.0 \pm 6.5$ & $98.9 \pm 2.1$ \\
\hline FEF $25 \%-75 \%$ before bronchoscopy $\%$ of baseline & $99.4 \pm 0.5$ & $60.5 \pm 9.8$ & $97.4 \pm 0.8$ \\
\hline \multicolumn{4}{|l|}{$\begin{array}{l}\text { Immunosuppression at the time of bronchoscopy } \\
n \text { of patients treated with }\end{array}$} \\
\hline Cyclosporin A/Azathioprin/Prednisolon & 5 & 1 & 0 \\
\hline Cyclosporin A/Mycophenolatmofetil/Prednisolon & 2 & 0 & 0 \\
\hline Tacrolimus/Azathioprin/Prednisolon & 0 & 3 & 8 \\
\hline
\end{tabular}

Data are presented as mean \pm SEM unless otherwise stated. LT: lung transplantation; BOS: bronchiolitis obliterans syndrome; F: female; M: male; FEV1: forced expiratory volume in one second; FEF $25 \%-75 \%$ : forced midexpiratory flow. 
Table 2. - Cell differentials of bronchial brush biopsies

\begin{tabular}{lccccccc}
\hline & $\begin{array}{c}\text { Subjects } \\
\mathrm{n}\end{array}$ & $\begin{array}{c}\text { Cell } \\
\text { count } 10^{6}\end{array}$ & $\begin{array}{c}\text { Epithelials } \\
\%\end{array}$ & $\begin{array}{c}\text { Macrophages } \\
\%\end{array}$ & $\begin{array}{c}\text { Neutrophils } \\
\%\end{array}$ & $\begin{array}{c}\text { Lymphocytes } \\
\%\end{array}$ & $\begin{array}{c}\text { Eosinophils } \\
\%\end{array}$ \\
\hline$<3$ months & 7 & $0.6 \pm 0.2$ & $89 \pm 4$ & $6 \pm 3$ & $3 \pm 1$ & $0 \pm 0$ & $0 \pm 0$ \\
BOS & 4 & $2.9 \pm 0.4$ & $79 \pm 11$ & $5 \pm 3$ & $15 \pm 12$ & $1 \pm 1$ & $0 \pm 0$ \\
Non-BOS & 8 & $1.6 \pm 0.3$ & $95 \pm 2$ & $3 \pm 1$ & $1 \pm 1$ & $0 \pm 0$ & $0 \pm 0$ \\
\hline
\end{tabular}

Data are presented as mean \pm SEM. BOS: bronchiolitis obliterans syndrome.
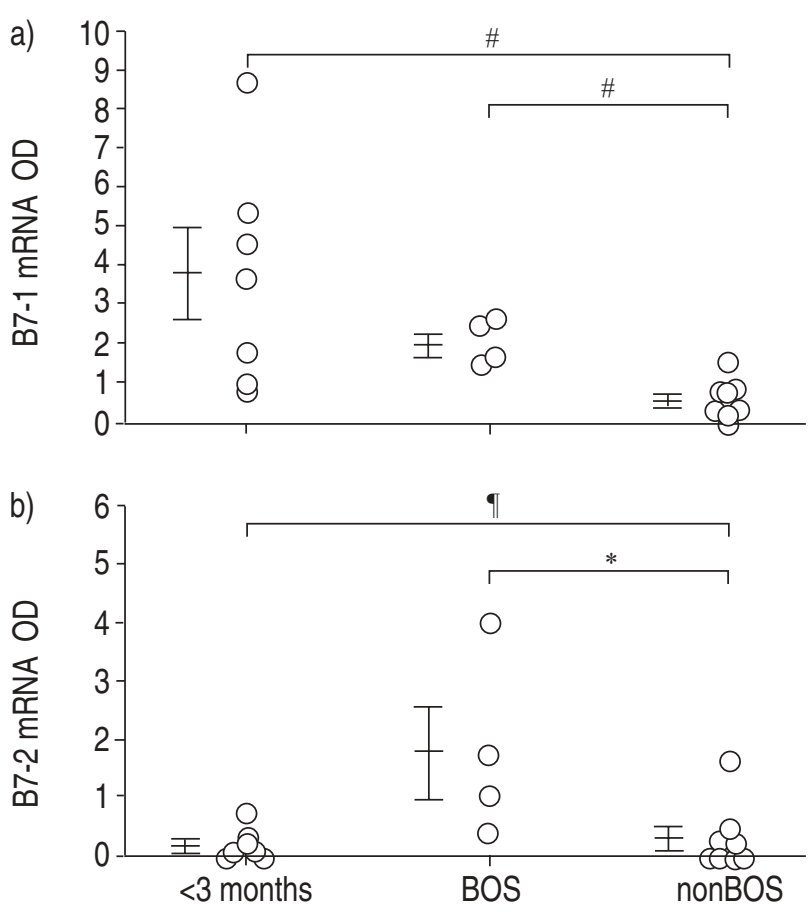

Fig. 1.-a) B7-1 and b) B7-2 messenger ribonucleic acid (mRNA) levels of cells harvested by bronchial brush biopsies. BOS: bronchiolitis obliterans syndrome; OD: optical density. *: $\mathrm{p}<0.05$; \#: $\mathrm{p}<0.005 ;{ }^{\uparrow}: \mathrm{p}=0.955$.

the linearity of the mRNA amplification had been determined and ranged from $37-45$ cycles (data not shown). Products were electrophoresed on a $1 \%$ agarose gel and viewed using a $300 \mathrm{~nm}$ ultraviolet (UV) transilluminator (Cybertech, Berlin, Germany). Samples from reverse transcription reactions, which did not contain reverse transcriptase, served as negative controls. For quantification, PCR bands were stained with ethidium bromide (Sigma, Munich, Germany) and scanned with a UV densitometer (Cybertech). The intensity of the B7-1 and B7-2 bands was corrected for the housekeeping gene GAPDH. To confirm that the correct PCR products had been amplified, the gel bands were excised from the gel and sequenced (TopLab, Martinsried, Germany).

\section{Statistics}

All data are expressed as the mean \pm SEM. Data were compared for significant differences using the MannWhitney rank sum test.

\section{Results}

\section{Cell differentials of bronchial brush specimens}

The return of bronchial epithelial cells using the technique of bronchial brushing ranged from $79 \%$ in BOS patients to $95 \%$ in patients without BOS and the number of contaminating macrophages was low (3-6\%) (table 2). More neutrophils (15\%) were harvested in the BOS patients compared to the group of patients studied in the first 3 months after transplantation $(3 \%)$ and patients without BOS $(1 \%)$, respectively. This interesting finding explains the slightly lower relative yield of bronchial epithelial cells in the BOS patients.

B7-1 and B7-2 messenger ribonucleic acid levels of cells harvested by bronchial brush biopsies

The B7-1 mRNA levels of both the group studied $<3$ months after LT and the BOS group were significantly elevated compared to the non-BOS patients $(\mathrm{p}<0.005$ for each comparison). The B7-2 mRNA levels of the BOS group surpassed the non-BOS, with the differences reaching statistical significance $(\mathrm{p}<0.05$ for each comparison) (fig. 1).

\section{Cell differentials of bronchoalveolar lavage fluid samples}

Macrophages represented the predominant cell type in the three groups of patients studied (63-87\% of total cells) (table 3 ). Characteristically for BOS patients, a

Table 3. - Cell differentials of and bronchoalveolar lavage fluid samples

\begin{tabular}{lcccccc}
\hline & Subjects n & Cell count $10^{6}$ & Macrophages \% & Neutrophils \% & Lymphocytes \% & Eosinophils \% \\
\hline$<3$ months & 7 & $6.5 \pm 1.2$ & $85 \pm 3$ & $5 \pm 1$ & $8 \pm 2$ & $2 \pm 1$ \\
BOS & 4 & $24.6 \pm 10.8$ & $63 \pm 19$ & $31 \pm 21$ & $6 \pm 4$ & $0 \pm 0$ \\
non-BOS & 8 & $14.8 \pm 2.9$ & $87 \pm 6$ & $11 \pm 6$ & $2 \pm 1$ & $0 \pm 0$ \\
\hline
\end{tabular}

Data are presented as mean \pm SEM. BOS: bronchiolitis obliterans syndrome. 

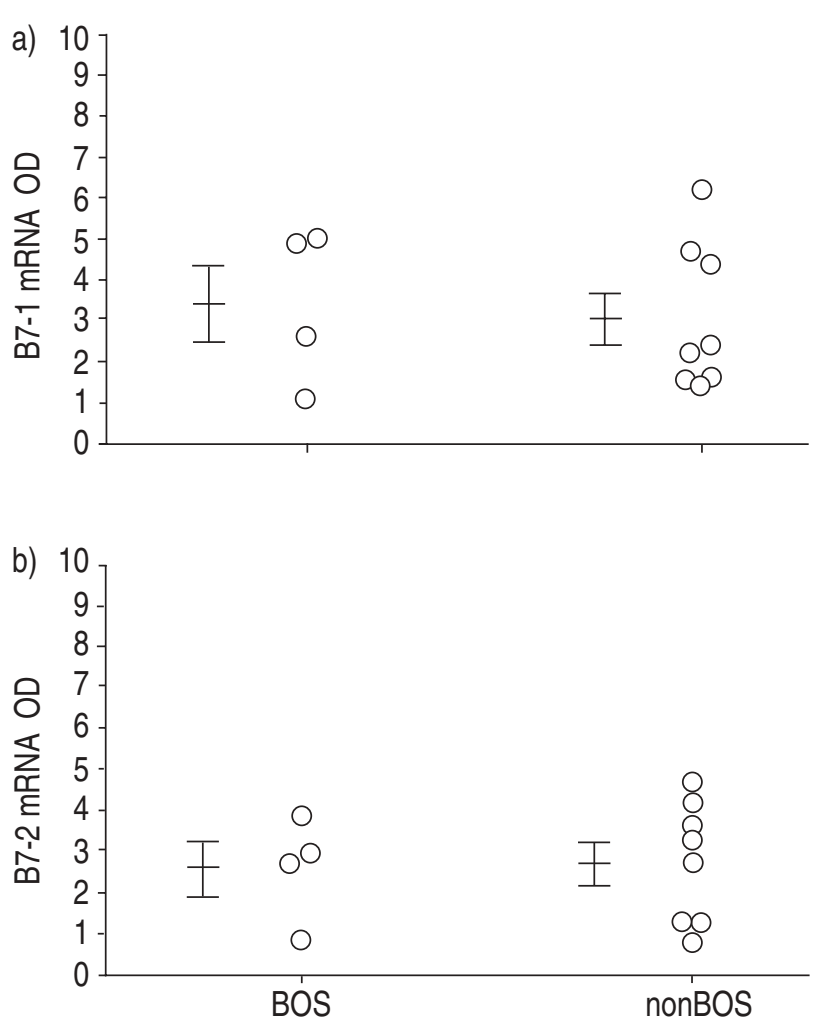

Fig. 2. -a) B7-1 and b) B7-2 messenger ribonucleic acid (mRNA) levels of bronchoalveolar lavage fluid cells in bronchiolitis obliterans syndrome (BOS) and non-BOS patients. OD: optical density. No significant differences were found $(\mathrm{p}=0.683$ for each comparison).

higher number of neutrophils (by absolute and relative counts) could be observed in BOS patients (31\% of total cells) compared to the group of patients studied in the first 3 months after LT $(5 \%)$ and patients without BOS $(11 \%)$.

\section{B7-1 and B7-2 messenger ribonucleic acid levels of bronchoalveolar lavage fluid cells}

No significant differences in the B7-1 and B7-2 mRNA expression of BALF cells was found $(\mathrm{p}=$ 0.683 for each comparison) (fig. 2).

\section{Discussion}

Significantly elevated B7-1 mRNA levels were found in bronchial epithelial cells from patients examined early after LT ( $<3$ months) compared to patients without evidence of LT dysfunction $>3$ months after LT. The group of patients with BOS showed significantly elevated levels of both costimulatory molecules B7-1 and B7-2 compared to the patients without evidence of LT dysfunction ( $>3$ months after LT). Considering that the predominant cells in normal and transplanted lungs from patients with and without BOS are macrophages, B7-1 and B7-2 mRNA were found in BALF cells as expected. Since no significant differences for B7-1 and
B7-2 mRNA between BOS patients and patients without evidence of LT dysfunction were found, the role of costimulation by alveolar macrophages in patients after LT remains unclear. Although statistically significant, at this point the finding of elevated levels of B7-1 and B7-2 mRNA in BOS patients has to be interpreted carefully because of the low number of patients in this group $(n=4)$.

Another limitation of the study is that the differences were shown only by the comparison of relative mRNA levels, and even if GAPDH is widely used and accepted as an internal standard in reverse transcriptase (RT)-PCR, a constant level of expression for this housekeeping gene cannot be guaranteed. It could be argued that theoretically the B7-1 and B7-2 mRNA detected by semiquantitative RT-PCR may be derived from contaminating macrophages in the bronchial brush specimens. However, there are several arguments against this assumption: 1) no correlation was found between the amount of macrophages harvested by brush biopsies and the message of B7-1 or B7-2; 2) significant amounts of B7-1 and B7-2 mRNA could be detected in samples where contamination of macrophages was not detectable; and 3) as PCR conditions (number of cycles, etc.) were the same for cells from bronchial brush specimens and BALF cells (mainly macrophages), such high levels of B7-1 and B7-2 from the low numbers (3-6\%) of contaminating macrophages would not be expected.

The patients studied here were clinically stable and had no evidence of acute allograft rejection, cytomegalovirus pneumonitis, bacterial or viral infections. Therefore, it remains unclear whether upregulation of costimulatory molecules on bronchial epithelial cells is specific to BOS or may also occur in a variety of other pathological conditions.

Interestingly in this study, idiopathic pulmonary fibrosis, a disease in which B7-1 and B7-2 expression in bronchiolar and alveolar epithelial cells has been described recently [6], was the underlying disease in five of the seven patients in the group examined early after LT ( $<3$ months). This group showed significantly elevated B7-1 mRNA levels compared to the non-BOS patients $(\mathrm{p}<0.005)$. Although unlikely, the fact that the elevated B7-1 mRNA levels were related to the pretransplant disease in these patients cannot be excluded.

It is well established that an efficient alloimmune response requires $\mathrm{T}$-cell activation and to achieve full activation engagement of costimulatory molecules like B7-1 and B7-2 in concert with MHC II is needed [1]. The importance of $\mathrm{B} 7-1$ and $\mathrm{B} 7-2$ is supported by animal and human studies, with the fusion protein CTLA-4-Ig blocking the B7-CD28 pathway. In a primate renal allograft model, CTLA-4-Ig was able to prevent and rescue allograft rejection [10]. In patients transplanted with histoincompatible bone marrow allografts, use of CTLA4-Ig to induce alloantigenspecific anergy resulted in a remarkably low incidence (three out of 11) of acute graft versus host disease [11]. Under normal circumstances the parenchymal donor cells lack B7 costimulatory molecules, and the indirect alloantigen presentation of the transplant recipient by 
professional APCs is believed to be the predominant pathway of T-cell activation in the long term after transplantation [1]. However, this concept is based mainly on experiments with rodents and does not take into account recent reports of B7 expression on human cells, which do not belong to the professional APCs, for example epithelial cells from different tissues including the lung [2-6]. In contrast to the expression of B7 molecules, human bronchial epithelial cells are well known to express MHC II antigens [12].

Before the discovery of costimulatory molecules, the finding of enhanced expression of MHC II molecules on bronchial epithelial cells in patients with $\mathrm{OB}$ after LT [13] as well as in animal lung allograft models [14] led to the hypothesis that OB is a rejection process initiated by and directed to the bronchial epithelium. The results presented here of elevated mRNA levels of B7-1 and B7-2 in patients with BOS shed new light on this hypothesis. It is as yet unclear how B7-1 and B7-2 expression may be regulated in epithelial cells. Generally cell-to-cell interactions, cytokines (e.g. interleukin-4, interferon- $\gamma$ ) or bacterial endotoxin are among the factors that control the regulation of $\mathrm{B} 7-1$ and B7-2 expression [15]. It could be speculated that contact of donor epithelium with recipient cells, repeated or latent viral infection (e.g. with cytomegalovirus), or bacterial infection and colonization (e.g. pseudomonas species) creates an inflammatory millieu within the airways that leads to upregulation of B7 molecules on epithelial cells.

In summary, the presented data support the hypothesis that bronchial epithelial cells may play an active immunological role in (chronic) lung allograft rejection. More investigation on the function of lung epithelial cells in the pathogenesis of obliterative bronchiolitis is needed.

\section{References}

1. Sayegh MH, Turka LA. Mechanisms of disease: the role of T-cell costimulatory activation pathways in transplant rejection. $N$ Engl J Med 1998; 339: 1813 1821.

2. Ye G, Barrera C, Fan X, et al. Expression of B7-1 and B7-2 costimulatory molecules by human gastric epithelial cells: potential role in $\mathrm{CD} 4+\mathrm{T}$ cell activation during Helicobacter pylori infection. J Clin Invest 1997; 99: 1628-1636.

3. Manoussakis MN, Dimitriou ID, Kapsogeorgou EK, et al. Expression of B7 costimulatory molecules by salivary gland epithelial cells in patients with Sjogren's Syndrome. Arthritis Rheum 1999; 42: 229-239.

4. Tsuneyama K, Harada K, Yasohima M, Kaji K,
Gershwin ME, Nakanuma Y. Expression of costimulatory factor B7-2 on the intrahepatic bile ducts in primary biliary cirrhosis and primary sclerosing cholangitis: an immunohistochemical study. J Pathol 1998; 186: 126-130.

5. Battifora M, Pesce G, Paolieri F, et al. B7-1 costimulatory molecule is expressed on thyroid follicular cells in Hashimoto's thyroiditis, but not in Graves' disease. Clin Endocrinol Metab 1998; 83: 4130-4139.

6. Kaneko Y, Kuwano K, Kunitake R, Kawasaki M, Hagimoto N, Hara N. B7-1, B7-2 and class II MHC molecules in idiopathic pulmonary fibrosis and bronchiolitis obliterans-organizing pneumonia. Eur Respir $J$ 2000; 15: 49-55.

7. Reichenspurner H, Girgis RE, Robbins RC, et al. Obliterative bronchiolitis after lung and heart-lung transplantation. Ann Thorac Surg 1995; 60: 18451853.

8. Cooper JD, Billingham M, Egan T, et al. A working formulation for the standardization of nomenclature and for clinical staging of chronic dysfunction in lung allografts. International Society for Heart and Lung Transplantation. J Heart Lung Transplant 1993; 12: 713-716.

9. Patterson GM, Wilson S, Whang JL, et al. Physiologic definitions of obliterative bronchiolitis in heart-lung and double lung transplantation: a comparison of the forced expiratory flow between $25 \%$ and $75 \%$ of the forced vital capacity and forced expiratory volume in one second. J Heart Lung Transplant 1996; 15: 175181.

10. Glanville AR, Tazelaar HD, Theodore J, et al. The distribution of MHC class I and II antigens on bronchial epithelium. Am Rev Respir Dis 1989; 139: 330-334.

11. Taylor PM, Rose ML, Yacoub MH. Expression of MHC antigens in normal human lungs and transplanted lungs with obliterative bronchiolitis. Transplantation 1989; 48: 506-510.

12. Pomaniuk A, Prop J, Petersen AH, Wildevuur CR, Nieuwenhuis P. Expression of class II major histocompatibility complex antigens by bronchial epithelium in rat lung allografts. Transplantation 1987; 44: 209-214.

13. Kirk AD, Harlan DM, Armstrong NN, et al. CTLA4$\mathrm{Ig}$ and anti-CD40 ligand prevent renal allograft rejection in primates. Proc Natl Acad Sci USA 1997; 94: 8789-8794.

14. Guinan EC, Boussiotis VA, Neuberg D, et al. Transplantation of anergic histoincompatible bone marrow allografts. $N$ Engl J Med 1999; 340: 1704-1714.

15. Lenschow DJ, Walunas TL, Bluestone JA. CD28/B7 system of $\mathrm{T}$ cell costimulation. Annu Rev Immunol 1996; 14: 233-258. 